\title{
Air-Steam Etched Construction of Hierarchically Porous Metal-Organic Frameworks
}

\author{
Hongliang Huang ${ }^{1}$, Yuxiu Sun ${ }^{1}$, Xuemeng Jia ${ }^{1}$, Wenjuan Xue ${ }^{1}$, Chenxu Geng ${ }^{1}$, Xin Zhao ${ }^{1}$, \\ Donghai $\mathrm{Mei}^{1}$, and Chongli Zhong ${ }^{1}$
}

${ }^{1}$ Tiangong University

October 21, 2020

\begin{abstract}
The introduction of mesoporosity into the microporous metal-organic frameworks (MOFs) is expected to expand their applications. Herein, we report a green and facile method to obtain hierarchically porous MOF structures by using an air-steam etching process. By virtue of the protonation reaction between the imidazole moiety and water vapor, the protonated imidazole related linkers leave the framework, resulting in the formation of mesopores in the zeolitic imidazolate frameworks (ZIFs), as exemplified by ZIF-8. Given the mild etching process, the materials' structural integrity and crystallinity are well maintained. Remarkably, the proposed steam etching approach is generally applicable, which can be readily extended to other ZIFs, such as ZIF-14, ZIF-69, and ZIF-71, thus representing a powerful strategy to construct hierarchically porous MOF materials.
\end{abstract}

\section{Hosted file}

Manuscript.pdf available at https://authorea.com/users/368968/articles/487948-air-steametched-construction-of-hierarchically-porous-metal-organic-frameworks 